\title{
4 years' experience of head and neck tuberculosis in a south London hospital
}

\author{
N Choudhury BSc MRCS ${ }^{1}$ G Bruch MD ${ }^{1}$ P Kothari FDSRCS ${ }^{1}$ G Rao FRCPath ${ }^{2}$ R Simo FRCS ${ }^{1}$
}

$J$ R Soc Med 2005;98:267-269

\section{SUMMARY}

In a south London department of otorhinolaryngology and head and neck surgery, 33 cases of tuberculosis were diagnosed in 4 years. The most common presentation was cervical adenitis (58\%) and in some cases the initial investigations suggested malignant disease. Most of the patients were of non-British origin but none proved to be HIV seropositive. Fine-needle aspiration was positive for tuberculosis in 7 of 19 patients. 21 patients required a surgical procedure for diagnosis.

\section{INTRODUCTION}

Tuberculosis (TB) is the world's leading cause of death from a single infective agent and a rising incidence has caused the World Health Organization to declare the disease a global emergency. ${ }^{1,2}$ Among the factors associated with the reversal of a previous decline are increased global travel and a rising prevalence of immunodeficiency through HIV infections or drug therapy. In the UK, pulmonary tuberculosis is the most common manifestation but extrapulmonary disease is likewise increasing. Here we review four years' experience of head and neck tuberculosis - a condition that can present diagnostic and therapeutic challenges.

\section{METHODS}

We retrospectively reviewed the case notes of all patients with proven extrapulmonary tuberculosis seen in the Otorhinolaryngology and Head and Neck Department of University Hospital Lewisham from December 1999 to January 2004. (All patients had then been referred to a TB clinic in the Department of Chest Medicine for further management.) Routine tests included full blood count, C-reactive protein and a chest X-ray. Patients with neck, thyroid and salivary gland masses underwent ultrasoundguided fine-needle aspiration, with cytological and microbiological examination including staining for acid-fast bacilli (Ziehl-Neelsen). Where tuberculosis was suspected, sputum samples were obtained for Ziehl-Neelsen staining.

Departments of ${ }^{1}$ Otorhinolaryngology and Head and Neck Surgery, and ${ }^{2}$ Microbiology, University Hospital Lewisham, London, UK

Correspondence to: Ms Natasha Choudhury, West Middlesex University Hospital, Twickenham Road, Isleworth TW7 6AF, UK

E-mail: natashamasood1@aol.com
In patients with inconclusive results further biopsies were taken for microbiological analysis, including culture on Löwenstein-Jensen medium, and histological assessment. Patients with proven TB were subsequently counselled and tested for HIV at the chest clinic.

\section{RESULTS}

33 patients were seen over the study period, 21 male 12 female, mean age 40 years (range $21-75$ ). 15 had a history of previous TB contact. 18 were of South Asian origin and only 'Caucasian'. In 19 patients (58\%), the presenting feature was cervical lymphadenitis. In addition, 9 had salivary gland TB, 2 laryngeal $\mathrm{TB}$, and 1 each nasopharyngeal, hypopharyngeal and ear TB. 21 patients required some sort of surgical procedure for diagnosis (Figure 1). In 12 patients the disease was producing constitutional symptoms such as fever, weight loss or night sweats. None of the patients proved to be HIV positive. 16 had evidence of TB on their chest X-ray.

The modes of microbiological and histopathological assessment were Ziehl-Neelsen stain 12/33, Mycobacterium tuberculosis culture 18/29 and histopathology 21/21. No patient had an atypical mycobacterial infection but in 2 the organism was resistant - in one case to streptomycin and in the other to isoniazid.

To highlight some of the diagnostic dilemmas we describe three of the cases.

\section{Case 1}

A man of 32 sought advice after two weeks with an enlarging neck mass and hoarseness. The mass was hard and fixed, measuring $6 \mathrm{~cm} \times 6 \mathrm{~cm}$, and he had a right vocal cord paresis. The mass was sampled by fine-needle aspiration which yielded no help. CT showed a large necrotic mass in 


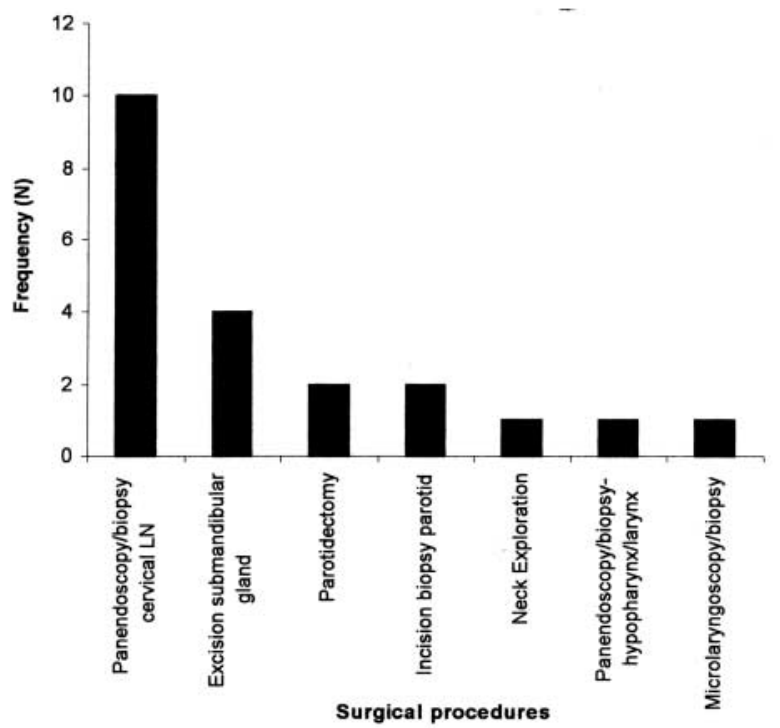

Figure 1 Surgical procedures performed to obtain histological diagnosis of TB

the right side of the neck (Figure 2) and also an apical right lung mass; the appearance suggested a malignant Pancoast type tumour (Figure 3). Bronchoscopy and lavage did not confirm this suspicion. A subsequent Heaf test was strongly positive (grade 4). Incision biopsy of the neck mass then yielded material that was positive for TB on microscopy, culture and histology.

\section{Case 2}

A man of 45 reported six months of dysphagia and odynophagia and four months of anorexia, weight loss, night sweats and dysphonia. At initial oesophagogastroduodenoscopy, gastroenterologists identified a large abnormal supraglottic and hypopharyngeal mass that was suspicious of carcinoma; however, multiple gastric biopsies showed no

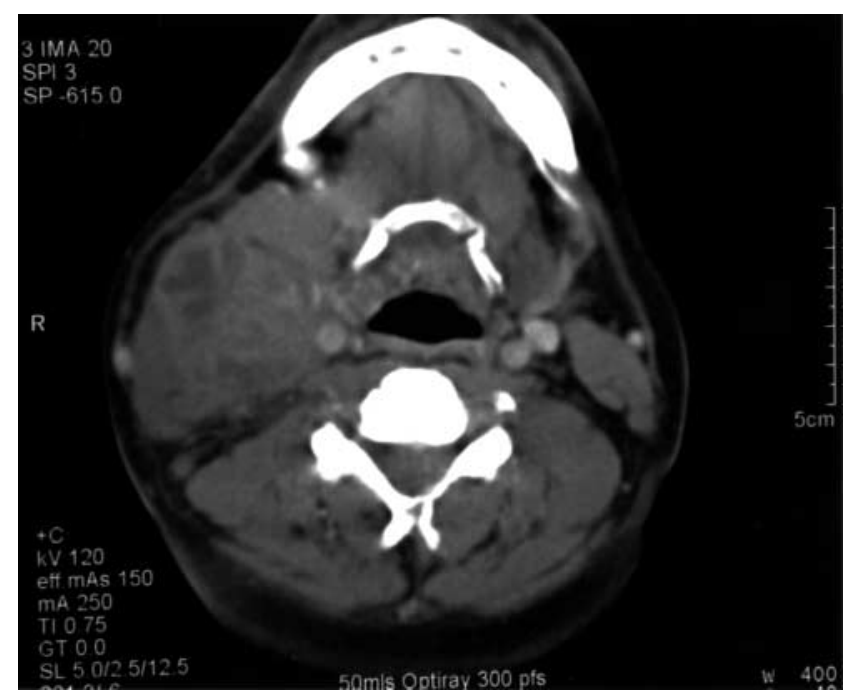

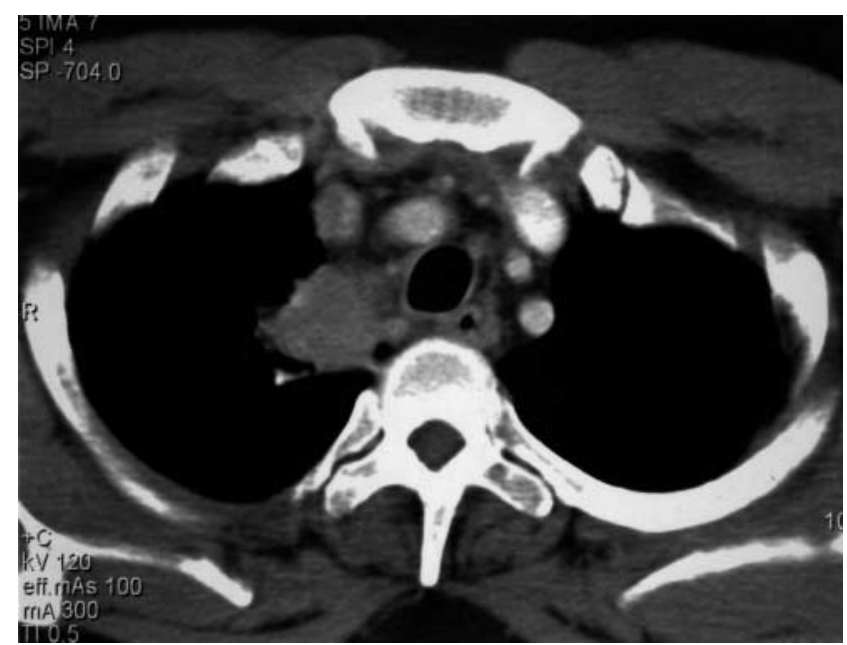

Figure $3 \mathbf{C T}$ showing apical mass in right lung, case 1

evidence of metaplasia, dysplasia or malignancy. On referral to our department he underwent panendoscopy and biopsy of the supraglottic and piriform sinus mass. Hypopharyngeal and laryngeal TB was evident on microscopy, culture and histology. He also had pulmonary $\mathrm{TB}$, with a positive sputum smear and culture. Initially nasogastric feeding was required because of his rigid larynx, but he eventually recovered.

\section{Case 3}

A man aged 63 reported the sudden onset of unilateral hearing loss, tinnitus and acute vertigo. His pure-tone audiogram showed a $70 \mathrm{~dB}$ left sensorineural hearing loss. He had also been troubled by headaches and nausea, ataxia and dysarthria. CT showed mild hydrocephalus and cerebrospinal fluid was positive for $\mathrm{TB}$ on staining and culture. MRI identified a large cerebellar abscess that was likewise due to TB. Twelve months' therapy was initially recommended, but the treatment was extended to 2 years owing to disease reactivation.

\section{DISCUSSION}

In $\mathrm{UK}$ inner-city areas such as ours, the resurgence of TB is most evident in the growing immigrant population. ${ }^{1}$ Most of our 33 patients were from South Asia.

HIV infection is a risk factor, but none was found in the present series. Our experience of extrapulmonary TB is, however, typical in showing a high rate of cervical adenitis. $^{3,4}$ Historically, tuberculous cervical lymphadenitis has been more common in children and young adults but the peak age range has now shifted to $20-40$ years. $^{5}$

In such cases, clinical differentiation from lymphoma or secondary metastasis can be difficult. The simplest initial investigation is fine-needle aspiration cytology, but in our series this was positive for acid-fast bacilli in only 7 of 19 patients. Definitive diagnosis depends on a combination of 
microbiology and histology. Single-organ TB (e.g. salivary gland) can be particularly challenging to diagnose, and 21 of our patients (61\%) required some form of surgical procedure for diagnosis, including superficial parotidectomy and submandibular gland excision.

Conventional techniques for isolation and identification of $\mathrm{TB}$ can be time-consuming and delay treatment. Molecular investigation by means of the polymerase chain reaction allows rapid diagnosis and subtyping of the mycobacteria, ${ }^{6,7}$ and an even quicker technique with a specific DNA-probe can be applied to the small clinical specimen collected by fine-needle aspiration. ${ }^{8}$ However, these techniques are not yet routinely available in most UK otolaryngology and head and neck centres.

The present series illustrates some of the diagnostic dilemmas presented by extrapulmonary TB. Differentiation from neoplastic or other inflammatory conditions can be difficult, particularly in mucosal TB involving the larynx, hypopharynx and nasopharynx. Within our patient cohort, the original suspected diagnosis included nasopharyngeal carcinoma and an occult primary tumour. Conversely, we had a patient with a suspected recurrence of cervical TB that proved to be a lymphoma. These patients need the services of a multidisciplinary team of surgeons, chest physicians, microbiologists and community nurses.

\section{REFERENCES}

1 Williams R, Douglas-Jones T. Mycobacterium marches back. J Laryngol Otol 1995; 109:5-13

2 Nakajima H. Tuberculosis: a global emergency. World Health 1993;46:3

3 Artenstein $\mathrm{AW}$, Kim JH, Williams WJ, et al. Isolated peripheral tuberculous lymphadenitis in adults: current clinical and diagnostic issues. Clin Infect Dis 1995;20:876-82

4 Kourbeti IS, Maslow MJ. Non-tuberculous mycobacterial infections of the lung. Curr Infect Dis Resp 2000;2:193-200

5 Perlman DC, D'Amico R, Salomon N. Mycobacterial infections of the head and neck. Curr Infect Dis Rep 2001;3:233-41

6 Smith KC, Starke JR, Eisenach K, et al. Detection of Mycobacterium tuberculosis in clinical specimens from children using polymerase chain reaction. Pediatrics 1996;97:155-60

7 Totsch M, Bocher W, Brommelkamp E, et al. Diagnostic value of different PCR assays for the detection of mycobacterial DNA in granulomatous lymphadenopathy. J Pathol 1996;178:221-6

8 Yokoyama J, Shiga K, Saijo S, Matumoto K. Rapid diagnosis of cervical tuberculous lymphadenitis by application of DNA probe. Otol-Head Neck Surg 1999;121:501-4 